\title{
Game and Decision of the Developer and the Retailer under Similar Consignment Mode
}

\author{
Lei Jiang $^{+1}$, Wei Chen ${ }^{2}, \mathrm{Wu} \mathrm{Su}^{2}$ \\ ${ }^{1}$ Department of Mathematics, Chengdu Textile College, Chengdu, China \\ ${ }^{2}$ Department of Fundamental Courses, Chengdu Polytechnic, China
}

\begin{abstract}
Under the similar consignment background, this paper discusses the problem of how the supply chain made up of a developer and a retailer can make the optimal decision and coordinate the supply chain through bargaining. The retailer decides the proportion of the developer to carry out the product consignment, and the developer makes the product price and the product quality investment. The market demand depends on the product price and quality input of the developer, Different from the uncertain demand of consignment mode, in the situation which the market demand is certain, The game equilibrium solution under decentralized decision and the profit of supply chain under centralized decision are discussed .As a result, there is only a R-Stackelberg equilibrium in the decentralized decision, and the supply chain performance is better than the centralized decision. Finally, through the bargaining question, we get the motivation of the supply chain enterprises to adopt the centralized decision under the certain condition.
\end{abstract}

Keywords: consignment; centralized decision; supply chain coordination; Stackelberg game; bargaining

\section{Introduction}

In the field of supply chain management academic and industry, the research of consignment contract and consignment stock has been the hot field. Jun Ru and Yunzeng Wang [1] have discussed who should control inventory in the supply chain under consignment contract, but they don't study the supply chain coordination. The literature [2-4] are focused on consignment stock in the case of VMI, but they do not carry out the game analysis of supply chain. There are a lot of research results [5-7] in the research of consignment contract, these studies have taken into account more supply chain costs, such as the shortage cost and the inventory cost, etc. Avinadav [8] and Chernonog [9] have considered a consignment contract for a special product (virtual product), in this consignment model, only the developer's cost to product inputs is considered. The research background of all the above consignment models is based on the uncertainty of market demand.

Similar to Avinadav and Chernonog [8-9], the product in this article has sufficient capacity to ensure consumer demand. Therefore, for the sale of such products, it is not necessary to consider the shortage cost and the inventory cost. For this kind of special product, we also consider only one product cost in the discussion of this model. In this paper, a decision model similar to consignment mode is established by considering the supply chain game, and the corresponding mathematical model is established by combining consignment model with a special products. At the same time, the related problems are studied, which is the main content. Different from the traditional consignment model, basing on the assumption that the market demand is deterministic, this paper further analyze the optimal decision of the developer and the retailer, while the supply chain performance analysis and coordination problems are discussed.

\footnotetext{
+ Corresponding author. Tel.: + 8613882025709; fax: +86-28-87840049.

E-mail address: roraldo9@163.com.
} 
The remainder of this paper is organized as follows: The assumptions and the discussion of the basic model of supply chain is established in Section II; In Section III, we analyze the model and get the equilibrium results and the profit in R-Stackelberg game and centralized decision, respectively; Section IV provides a bargaining problem to investigate the profit distribution between the developer and retailer, so as to coordinate the supply chain; Section $\mathrm{V}$ summarizes the conclusions from the research and suggests future research directions.

\section{The Problem and Assumptions}

In this paper, the supply chain model of a special product is considered, which consists of a retailer and a developer. After the developer develops the product, the product is delivered to the retailer for sale, and the consumer purchases the desired product through the retailer building platform. In such this product sales mode, the developer first develop products, and at the same time, according to market demand, give the selling price of products; retailers provide the sales platform for products, and extract corresponding sales according to the regulations. The unit sales price of the product is $p$, the quality investment of the developer for the product is $x$, and the retailer divides the product of each unit of products into $t$. Meanwhile, we assume that the market demand is determined by the price of the product and the input of the developer to the product, and is certain. By the nature of the market demand function, it is easy to know that $D=D(p, x)$ is a decreasing function of $p$, which is the increasing and concave function of $x$. We continue to assume that demand is a product function of product price and quality investment.

$$
D(p, x)=\alpha(p) \beta(x)
$$

where $\alpha(p)$ is the monotone decreasing function of $p$, and $\beta(x)$ increases monotonously with $x$ and is the concave function of $x$.In order to analyze the structure of the equilibrium analytic solution of the problem and without losing generality, the demand price function is further assumed $\alpha(p)=a-b p$. At the same time, this paper assumes that the product's quality investment function $\beta(x)=c \sqrt{x}$ (the increasing and concave function). Therefore, the demand function of the product is :

$$
D(p, x)=(a-b p) c \sqrt{x}
$$

where $a, b, c$ are the constant greater than zero, indicates the potential demand of the market when the price is zero, and indicate the sensitivity of demand to price and the developer quality investment for product, respectively. To ensure the market demand $D(p, x)>0$, it must satisfy the requirement of $\alpha(p)=a-b p>0$, that is, $p<\frac{a}{b}$.Similar to the reference [8-9], because of the characteristics of their products, there have sufficient capacity to ensure that the needs of all consumers, so ,there will be no shortage cost; at the same time, the products that are not sold will not have the same inventory cost as solid products . Accordingly, for this kind of special products, it is different from the sale of the real product, and it does not need to consider the stock cost and the shortage cost. Based on the above reasons, only a cost consideration in the discussion of the model, product quality is the investment cost for developers of product development, which called the product cost of the investment. At the same time, the developer and the retailer are rational, all information is complete and there is not any private information.

\section{The Model and Analysis}

In order to better analyze the above problems, we discuss the equilibrium and optimal solution of each decision variable under decentralized and centralized decision, respectively.

\subsection{Decentralized Decision}

\section{a) dominated by the retailer}

In the decentralized decision situation, it is assumed that the retailer is the dominant, the retailer in the downstream of the supply chain and the developer in the upstream of the supply chain play a dynamic 
Stackelberg game, which is recorded as the R-Stackelberg game. The retailer is the leader of the game and the developer is the follower. In this case, the order of the game between the retailer and the developer is: before the product is sold, the retailer first announced the proportion of its sale products, the developer can get their own share based on the retailer's share, and make a corresponding decision on the sale price of the product and the cost of the product quality.

According to (1) and (2), the corresponding profits for the retailer and the developer are as follows:

$$
\begin{gathered}
\prod_{r}(t)=t p(a-b p) c \sqrt{x} \\
\prod_{d}(t)=(1-t) p(a-b p) c \sqrt{x}-x
\end{gathered}
$$

On the basis of (3) and (4), the equilibrium solutions of this game are solved by backward induction, and the optimal equilibrium decision lead by the retailer is represented by the subscript. The following theorems can be obtained.

Theorem1. If $b^{2}+8 a^{2}-12 a b<0, t_{r s}{ }^{*}=\frac{1}{2}, p_{r s}{ }^{*}=\frac{a}{2 b}, x_{r s}{ }^{*}=\frac{1}{256}\left(c \frac{a^{2}}{b}\right)^{2}$ are R-Stackelberg equilibrium solution.

Proof of Theorem1. Using the backward induction of Stackelberg game, the best reaction function of the developer is found first. According to (4), it is easy to get:

$\frac{\partial \mathrm{I}_{d}}{\partial p}=(1-t) c \sqrt{x}(a-2 b p) ; \frac{\partial \prod_{d}}{\partial x}=0.5 c(1-t) x^{-\frac{1}{2}}(a-b p)-1$.we let $\frac{\partial \mathrm{I}_{d}}{\partial p}=0$ and $\frac{\partial \mathrm{II}_{d}}{\partial x}=0$, respectively.

The response function to maximize the developer's profit is as follows: $p(t)=\frac{a}{2 b}$; $x(t)=\frac{1}{64}\left(c(1-t) \frac{a^{2}}{b}\right)^{2}$.Under this response function, the retailer's profit is $\prod_{r}(t)=\frac{1}{32} c^{2} t(1-t) \frac{a^{4}}{b^{2}}$.

The retailer maximized its profits to meet the first order condition. We let $\frac{\partial \prod_{r}}{\partial t}=\frac{1}{32} c^{2} \frac{a^{4}}{b^{2}}(1-2 t)=0$ and can get $t=\frac{1}{2}$.Meanwhile, $\frac{\partial^{2} \prod_{r}}{\partial^{2} t}=-\frac{1}{16} c^{2} \frac{a^{4}}{b^{2}}<0$, it satisfies the second order condition, that is, $1 \mathrm{I}_{r}$ is always the concave function with respect to $t$.Therefore, the retailer's optimal decision is $t_{r s}{ }^{*}=\frac{1}{2}$. We can also get the following formula in term of (4).

$$
\text { Since } \frac{\partial^{2} \prod_{d}}{\partial p^{2}}=-2 b(1-t) c x^{\frac{1}{2}}<0 ; \frac{\partial^{2} \prod_{d}}{\partial p \partial x}=\frac{1}{2}(1-t) c(a-2 b p) x^{-\frac{1}{2}} ; \frac{\partial^{2} \prod_{d}}{\partial x^{2}}=-\frac{1}{4}(a-b p)(1-t) c x^{-\frac{3}{2}},
$$

We further assume that the Hessian matrix of $\mathrm{I}_{d}(p, x)$ is $\mathrm{H}$. Its determinant is

$$
|H|=\frac{\partial^{2} \prod_{d}}{\partial p^{2}} \frac{\partial^{2} \prod_{d}}{\partial x^{2}}-\left(\frac{\partial^{2} \prod_{d}}{\partial p \partial x}\right)^{2}=\frac{1}{4}(1-t)^{2} c^{2} x^{-1}\left[4 b^{2} p^{2}+p\left(2 b^{2}-4 a b\right)-a^{2}+2 a b\right]
$$

Since $0<1-t<1$ and $c>0$, the developers' input to the product $x>0$. We can get:

When $4 b^{2} p^{2}+p\left(2 b^{2}-4 a b\right)-a^{2}+2 a b>0,|H|>0$.

So, the Hessian matrix of $\mathrm{II}_{d}(p, x)$ is negative and there must be a maximum .

If $4 b^{2} p^{2}+p\left(2 b^{2}-4 a b\right)-a^{2}+2 a b>0$, it must satisfy $\Delta=\left(2 b^{2}-4 a b\right)^{2}-4 \times 4 b^{2} \times\left(-a^{2}+2 a b\right)<0$.

It can be obtained after the solution is simplified as follows: $b^{2}+8 a^{2}-12 a b<0$.

If the condition is satisfied, we substitute $t_{r s}{ }^{*}=\frac{1}{2}$ into $p(t)$ and $x(t)$, respectively. The developer's optimal decisions can obtained.

This completes the proof of Theorem1. 
According to Theorem 1, the following proposition is easily obtained .

Proposition 1. In the R-Stackelberg game case, the profits of the developer and the retailer are obtained as follows: $\prod_{r}^{*}=\frac{1}{128}\left(c \frac{a^{2}}{b}\right)^{2} ; \prod_{d}^{*}=\frac{1}{256}\left(c \frac{a^{2}}{b}\right)^{2}$.

Proof of Proposition 1. After substituting $t_{r s}{ }^{*}, p_{r s}{ }^{*}, x_{r s}{ }^{*}$ into (3) and (4), We can calculate the profits of the developer and the retailer in the R-Stackelberg game case.

By analyzing and comparing the optimal decision and the profits of upstream and downstream enterprises under the situation which the retailer is leader, the following proposition can be obtained accordingly .

Proposition 2 i) $t_{r s}{ }^{*}$ is independent of all demand sensitivity coefficient, $p_{r s}{ }^{*}$ is only independent of the sensitivity coefficient ; ii) $\Pi_{r}^{*}=2 \Pi_{d}{ }^{*}$. It is easy to get the conclusion by Theorem 1 and proposition 1 .

Proposition 2 indicates that, in the decentralized decision case, when the retailer is the leader of the supply chain, no matter how the market demand changes with sensitivity, the developer and the retailer will always share half of the profits in order to ensure their respective best profits and stabilize the supply chain. Simultaneously, regardless of the developer's market sensitivity to product investment, the developer does not change the product price. As the leader, facing the product sales, the retailer has more profit than the developer, twice as much as the developer.

\section{b) dominated by the developer}

Unlike the previous case, the upstream developer is considered to be the leader of the supply chain; The retailer is the follower and makes the corresponding decision with the decision of the developer, and they play Stackelberg game. This game is recorded as D-Stackelberg game. At this time, the order of the game is: before the product is market-oriented, the developer first gives the selling price of the product and the investment of product quality. The retailer declare their share of product sales after observing the price and quality investments given by developers. Similar to the R-Stackelberg game, the profits of the developer and the retailer are still (3) and (4), respectively.

Similar to the front analysis, the equilibrium decision is depicted by the subscript $\&$, when the developer is leader. By calculation, the following theorem can be obtained.

Theorem 2. In the D-Stackelberg game case, there is no equilibrium solution.

Proof of Theorem 2. The backward induction method is used to solve the response function of the optimal retailer. It is easy to get that the solution of $t$ is 1 . However, $\frac{\partial^{2} \Pi_{r}}{\partial t^{2}}=0$, it does not satisfy the concave property and there is no extreme value, so there is no D-Stackelberg equilibrium solution in the game.

Theorem 2 tell us, When the developer foresees that the retailer makes a decision on $t=1$, it is bound to make a decision not to invest in the product. At the same time, the pricing strategy for the goods is: either to price at $p=1$, or to price at $p=a / b$. This will lead to the market demand is zero, and the retailer have no profits. So, in this game, there is no equilibrium decision. Of course, this also reflects a real situation: in the real market, for the sale of such consignment goods, the retailer often has the initiative to become a strong side, and the developer tends to become the follower, such as network sales platforms, etc.

\subsection{Centralized Decision}

In centralized decision case, the developer and the retailer form a whole, belonging to an enterprise to make decisions on the selling price of the products and the quality investment of the products, so as to reach the optimal profit of the whole supply chain system. Accordingly, the profit of the whole supply chain is:

$$
\prod_{d+r}=\prod_{r}+\prod_{d}=p(a-b p) c \sqrt{x}-x
$$

We assume that the optimal solution is $p^{\&}$ and $x^{\&}$. Then, $p^{\&}$ and $x^{\&}$ must be meet: 


$$
\left\{\begin{array}{c}
\frac{\partial \mathrm{I}_{d+r}}{\partial p}=c(a-b p) \sqrt{x}=0 \\
\frac{\partial \prod_{d+r}}{\partial x}=\frac{1}{2} c p(a-b p) x^{-\frac{1}{2}}=0
\end{array}\right.
$$

Meanwhile, we can get:

$$
\frac{\partial^{2} \prod_{d+r}}{\partial p^{2}}=-2 b \sqrt{x}<0 ; \frac{\partial^{2} \prod_{d+r}}{\partial p \partial x}=\frac{c}{2}(a-2 b p) x^{-\frac{1}{2}} ; \frac{\partial^{2} \prod_{d+r}}{\partial x^{2}}=-\frac{1}{4} c p(a-b p) x^{-\frac{3}{2}} \text {.So, similar to the }
$$

proof of Theorem 1, we can still get: when $b^{2}+8 a^{2}-12 a b<0$, the Hessian matrix of $1 \mathrm{I}_{d}(p, x)$ is negative, $\mathrm{Il}_{d}(p, x)$ is a joint concave function on $p$ and $x$.We can get the following conclusion.

Theorem 3. i) If $b^{2}+8 a^{2}-12 a b<0$, the optimal solution of the developer and the retailer under centralized decision case is, respectively. $p^{\&}=\frac{a}{2 b} ; x^{\&}=\frac{1}{64}\left(c \frac{a^{2}}{b}\right)^{2}$; ii) The maximum profit of the whole supply chain is, $\Pi_{d+r}\left(p^{\&}, x^{\&}\right)=\frac{1}{64}\left(c \frac{a^{2}}{b}\right)^{2}$.

Compared with the optimal decision and the profit under decentralized decision and centralized decision, we can obtain the following proposition.

Proposition 3. i) $p^{\&}=p^{*} ; x^{\&}=4 x^{*}$; ii) $\prod_{d+r}\left(p^{\&}, x^{\&}\right) \geq \prod_{r}\left(t^{*}\right)+\prod_{d}\left(p^{*}, x^{*}\right)$.

Proof of Proposition 3. i) Since $p^{\&}=\frac{a}{2 b} ; x^{\&}=\frac{1}{64}\left(c \frac{a^{2}}{b}\right)^{2} ; p_{r s}{ }^{*}=\frac{a}{2 b}, x_{r s}{ }^{*}=\frac{1}{256}\left(c \frac{a^{2}}{b}\right)^{2}$. We can obtain the corresponding conclusion. ii) Since $\prod_{r}\left(t^{*}\right)=\frac{1}{128}\left(c \frac{a^{2}}{b}\right)^{2} ; \prod_{d}\left(t^{*}\right)=\frac{1}{256}\left(c \frac{a^{2}}{b}\right)^{2}$ and $\prod_{d+r}\left(p^{\&}, x^{\&}\right)=\frac{1}{64}\left(c \frac{a^{2}}{b}\right)^{2}$, so, $\prod_{d+r}\left(p^{\&}, x^{\&}\right) \geq \prod_{r}\left(t^{*}\right)+\prod_{d}\left(p^{*}, x^{*}\right)$.

According to Proposition 3, we find that under the centralized decision, the developer will increase the investment in the quality of the product, which will increase the demand of the market, although the pricing has not changed. And the profit under the centralized decision is higher than the profit under the decentralized decision. Although the profit under the cooperative decision is optimal, not every member is optimal. Therefore, not every participant is motivated to adopt centralized decision. Therefore, in order to induce the members of supply chain to prefer the strategy under centralized decision. Instead of using the strategy of decentralized decision-making, we try to use bargaining problem[10-11] to distribute the extra profits and discuss how the developer and the retailer are more willing to make decision in centralized decision case so that the whole supply chain to achieve coordination.

\section{The Bargaining Problem}

The profit of supply chain in decentralized decision and centralized decision are respectively expressed as $\prod_{d+r}{ }^{*}$ and $\prod_{d+r}{ }^{\&}$. Substituting $p^{\&}$ and $x^{\&}$ into (3) and (4), respectively, we can get the profits of developers and retailers under centralized decision - making and the corresponding additional profits of the supply chain as follows:

$$
\begin{gathered}
\prod_{r}{ }^{\&}=\frac{1}{32} t\left(c \frac{a^{2}}{b}\right)^{2} \\
\prod_{d}^{\&}=\frac{1}{32}\left(\frac{1}{2}-t\right)\left(c \frac{a^{2}}{b}\right)^{2} \\
\Delta \prod_{d+r}=\prod_{d+r}{ }^{\&}-\prod_{d+r}^{*}
\end{gathered}
$$


In order to reasonably allocate extra profits, which makes both the developer and the retailer more willing to adopt centralized decision, the bargaining problem is:

$$
\left\{\begin{array}{l}
\Delta \prod_{r}=\prod_{r}{ }^{\&}-\prod_{r}{ }^{*} \geq 0 \\
\Delta \prod_{d}=\prod_{d}{ }^{\&}-\prod_{d}{ }^{*} \geq 0
\end{array}\right.
$$

where $\Delta \mathrm{II}_{d+r}=\Delta \mathrm{II}+\Delta \mathrm{II}_{d}$.

Theorem 4 If $\frac{1}{4} \leq t \leq \frac{3}{8}$, the developer and the retailer tend to adopt centralized decision, otherwise there will be incentives for decentralized decision.

Proof of Theorem 4. Combining Theorem 1 with (7) and (8), we can get: $\Delta \prod_{r}=\left(\frac{t}{32}-\frac{1}{128}\right)\left(c \frac{a^{2}}{b}\right)^{2}$;

$\Delta \prod_{d}=\left(\frac{1}{84}-\frac{8 t}{256}\right)\left(c \frac{a^{2}}{b}\right)^{2}$. Since $\frac{t}{32}-\frac{1}{128} \geq 0$ and $\frac{1}{84}-\frac{8 t}{256} \geq 0$, So, $\frac{1}{4} \leq t \leq \frac{3}{8}$.

We know, $\prod_{d+r}^{\&}=\prod_{d+r}\left(p^{\&}, x^{\&}\right)=\frac{1}{64}\left(c \frac{a^{2}}{b}\right)^{2} ; \prod_{d+r}{ }^{*}=\prod_{r}\left(t^{*}\right)+\prod_{d}\left(p^{*}, x^{*}\right)=\frac{3}{256}\left(c \frac{a^{2}}{b}\right)^{2}$.

By (10), it is easy to verify the formula: $\Delta \mathrm{I}_{d+r}=\Delta \mathrm{II}_{r}+\Delta \mathrm{I} \mathrm{I}_{d}$.

However, due to the lack of other information and more conditions, we cannot further study how to distribute the extra profits for the developer and the retailer, when coordinating the supply chain, in order to determine the profit distribution between the developer and the retailer, risk factors may are joined the decision makers, but the market demand which is considered in this paper is deterministic, we don't do much research anymore.

\section{Conclusion}

Based on the similar consignment mode of supply chain, this paper discusses the decentralized and centralized decision models of the developer and the retailer which the market demand is determinate. When the sensitivity coefficient of the demand function meets certain conditions, the corresponding optimal decision is obtained. It is found that the similar consignment model is only suitable for the R-Stackelberg game in the decentralized decision. It is also found that the whole profit of the supply chain in centralized decision is higher than the sum of the profit of the developer and the retailer in the decentralized decision. Finally, in order to coordinate the supply chain, the bargaining problem is adopted to get the incentive to adopt centralized decision when the retailer divides the income of the developer into a certain range. The next research direction is the game and coordination of the supply chain system when the demand is uncertain or adding the competition factor of the developer.

\section{Acknowledgements}

This paper is supported by foundation of Sichuan Educational Committee(17ZB0015) and foundation of Chengdu Textile College(2017fzlk04).

\section{References}

[1] Jun Ru,Yunzeng Wang. "Consignment contracting: Who should control inventory in the supply chain?". European Journal of Operational Research. 2010,201:760-769.

[2] Jun-Yeon Lee, Richard K. Cho. "Contracting for vendor-managed inventory with consignment stock and stockoutcost sharing". International Journal of Production Economics . 2014,151 (1) :158-173.

[3] Mehmood Khan,Mohamad Y. Jaber,Simone Zanoni, Lucio Zavanella. "Vendor managed inventory with consignment stock agreement for a supply chain with defective items".Applied Mathematical Modelling .2016, 40 (15-16): :7102-7114. 
[4] MY Jaber."A two-level supply chain with consignment stock agreement and stock-dependent demand".International Journal of Production Research .2015 , 53 (12) :3561-3572.

[5] Sijie Li, Zhanbei Zhu, Lihua Huang. "Supply chain coordination and decision making under consignment contract with revenue sharing". International Journal of Production Economics . 2009 , 120 (1) :88-99.

[6] Y Gerchak, Y Wang. "Revenue-Sharing vs. Wholesale-Price Contracts in Assembly Systems with Random Demand". Production \& Operations Management.2010, 13 (1) :23-33.

[7] Desheng Wu. "Coordination of competing supply chains with news-vendor and buyback contract". International Journal of Production Economics . 2013 , 144 (1) :1-13.

[8] Chernonog,T, Avinadav,T. "Price-setting under risk criteria for virtual products and services". European Journal of Operational Research. 2014,236:351-360.

[9] Tal Avinadav,Tatyana Chernonog."Consignment contract for mobile apps between a single retailer and competitive developers with different risk attitudes". European Journal of Operational Research.2015,246:949-957.

[10] Avinadav, T.,Herbon,A.,Spiegel,U. "Optimal inventory policy for a perishable item with demand function sensitive to price and time". European Journal of Operational Research.2013,144, 497-506.

[11]Xie, J. and J. C. Wei.2009. "Coordinating advertising and pricing in a manufacturer-retailer channel." European Journal of Operational Research.197 (2): 785-791. 